Revista Digital Universitaria

Vol. 21, Núm. 4, julio-agosto 2020

\title{
La investigación acerca del acoso y violencia escolares en México
}

\author{
Mercedes de Agüero Servín
}

\begin{abstract}
Resumen
Este artículo analiza la investigación publicada en español acerca del acoso y la violencia escolares en México; muestra los ejes temáticos de los estudios, y los tipos de violencia y de actores educativos implicados en los centros escolares de secundaria y bachillerato. Este texto ofrece un panorama introductorio acerca de cómo se expresan los actos de acoso, hostigamiento, discriminación y desigualdad, los cuales se reproducen en las relaciones sociales y construyen identidades, así como la manera en la que se vuelven prácticas sociales de la violencia. Estas prácticas generan aprendizajes sobre la convivencia escolar y construyen contextos e interacciones entre los actores educativos, que se traducen en un fenómeno histórico-cultural de reproducción, resistencia y conflicto de desigualdad social e inequidad.
\end{abstract}

Palabras clave: acoso, maltrato infantil, juventud, desigualdad social.

\section{Research on bullying and Violence at school in MeXico}

\begin{abstract}
This article analyzes the research published in Spanish about school bullying and school violence in Mexico; It shows the thematic axes of the studies, and the types of violence and educational actors involved in secondary and high schools. This text offers an introductory overview of how acts of bullyng, harassment, discrimination and inequality are expressed, they way they are reproduced in social relationships and build identities, as well as how they become social practices of violence. These practices generate learnings about school coexistence, and build contexts and interactions between educational actors, which translate into a historical-cultural phenomenon of reproduction, resistance and conflict of social inequality and inequity.
\end{abstract}

Keywords: bullying, child abuse, youth, social inequality.

DOI: http://doi.org/10.22201/cuaieed.16076079e.2020.21.4.2 


\section{Mercedes de Agüero Servín}

Subdirectora de Investigación en Educación, Coordinación de Universidad Abierta, Innovación Educativa y Educación a Distancia (CUAIEED).

Licenciada en Pedagogía por la Facultad de Filosofía y Letras de la Universidad Nacional Autónoma de México (UNAM), Mtra. En Investigación y Desarrollo de la Educación por la Universidad Iberoamericana, Ciudad de México y Dra. en Educación por la Universidad Autónoma de Aguascalientes. Se ha desempeñado como profesora de licenciatura, maestría y doctorado, y como investigadora de la educación en instituciones gubernamentales y universidades, tanto públicas y privadas, desde el año 1982 a la fecha. Está certificada como psicoterapeuta corporal Core Energética y Transpersonal, y tiene consulta clínica privada. Ha publicado numerosos artículos de investigación y divulgación en ciencias de la educación. Ha participado en varios libros y capítulos de libros sobre educación. 


\section{Introducción}

¿Alguna vez en la escuela, cuando fuiste niño o niña, tal vez entre los amigos, o jugando entre primos "te agarraron de bajada", "agarraron a alguien de su cochinito", "te traían asoleado", o "no dejaban en paz ni un segundo a tus amigos o hermanas"? Muchas veces pensamos que así son las cosas y que hay poco que hacer para que cambien las formas como nos hablamos, jugamos, nos comunicamos, trabajamos o convivimos en los espacios de vida cotidiana. Sin embargo, no deben ser así nuestras interacciones con otros, éstas pueden cambiar hacia un buen trato entre compañeros y amigos, en los espacios escolares y fuera de éstos.

Las relaciones entre quienes integran los distintos tipos de familias, los que conviven y trabajan en la escuela o en las oficinas, o entre las personas que intercambian servicios y productos, necesitan de un buen trato, ser amables, respetuosas, seguras, con formas de actuar y hablar confiables.

El trabajo y el juego son dos actividades primordiales en la vida de las personas, los espacios donde los realizamos son múltiples y diversos, y las maneras como hablamos e interactuamos con los demás también lo son. Cuando no actuamos con responsabilidad y no nos relacionamos pensando en el bien común y en una sensación de bienestar pueden existir distorsiones en las creencias y en las interrelaciones sociales, lo que puede generar uno o varios tipos de acoso y violencia en las escuelas. Estas relaciones perjudiciales se ejercen por personas cercanas y se padecen por quienes son importantes para nosotros, incluyéndonos, sin que podamos verdaderamente distinguir cómo es que se llegan a pasar los límites de la sana relación.

\section{La violencia escolar}

El objetivo de este artículo es compartir lo que se sabe acerca del acoso y hostigamiento escolares en México, a través de la investigación educativa en la literatura publicada en español. La investigación en ciencias sociales y de la educación es un potente medio para comprender, explicar y resolver problemas, y tomar decisiones fundamentales que permitan ofrecer mejores servicios educativos y elevar la calidad y el bienestar de los actores educativos. Permite fomentar la agencia de los estudiantes y el profesorado, así como el desarrollo humano y el aprendizaje.

Si los distintos actores educativos tomaran decisiones con base en estudios sustantivos, se podrían diseñar mejores contextos sociales y escolares para atender, prevenir y resolver de forma fundamental el acoso y la violencia, concebidos como un problema multidisciplinar y multifactorial. 
Los problemas de acoso escolar no ocurren en un coto de poder aislado del resto de las relaciones sociales que viven los jóvenes en secundarias y bachilleratos (Díaz-Barriga Arceo et al., 2019), sino que están en perfecta sintonía con el ambiente social, cultural y mediático con el que se relacionan.

Violencia, maltrato, acoso, intimidación, hostigamiento y acoso escolar son todos términos que se encuentran en la literatura especializada y científica en México. Si bien hay programas e información disponible para hacer frente a la problemática, tanto de educación básica como para educación media, las instituciones escolares se muestran desconcertadas ante dichos procesos sociales. A veces parecen sorprendidas, en otras su actuar es frívolo o superficial, y en muchas hay relaciones sociales que se hacen ciegas a lo que hacen los profesores y profesoras, estudiantes y trabajadores en general, comportamientos que las posicionan en procesos de complicidad.

Por ejemplo, los casos de acoso y abuso sexual en las escuelas, los de consumo y venta de drogas en las zonas circundantes a las secundarias y bachilleratos; las denuncias infructuosas de desaparición de niñas y adolescentes; y los casos más escandalosos, como la desaparición masiva de jóvenes, hombres y mujeres, en los que están implicados autoridades de gobierno de distintos niveles en colusión con policías municipales, estatales y federales, hasta con el ejército. Así, lejos de proponer una sociedad mejor a sus alumnos, las instituciones escolares reproducen automáticamente y fuera de toda conciencia las relaciones de poder que le circundan: las faltas de respeto, abusos, malos tratos, daños a la propiedad ajena, la ley del más fuerte, etcétera (Castillo Rocha y Pacheco Espejel, 2008, p. 840, ver video 1).

Video 1. Cambiar la educacion para cambiar el mundo: Claudio Naranjo (Dorado, 2019).

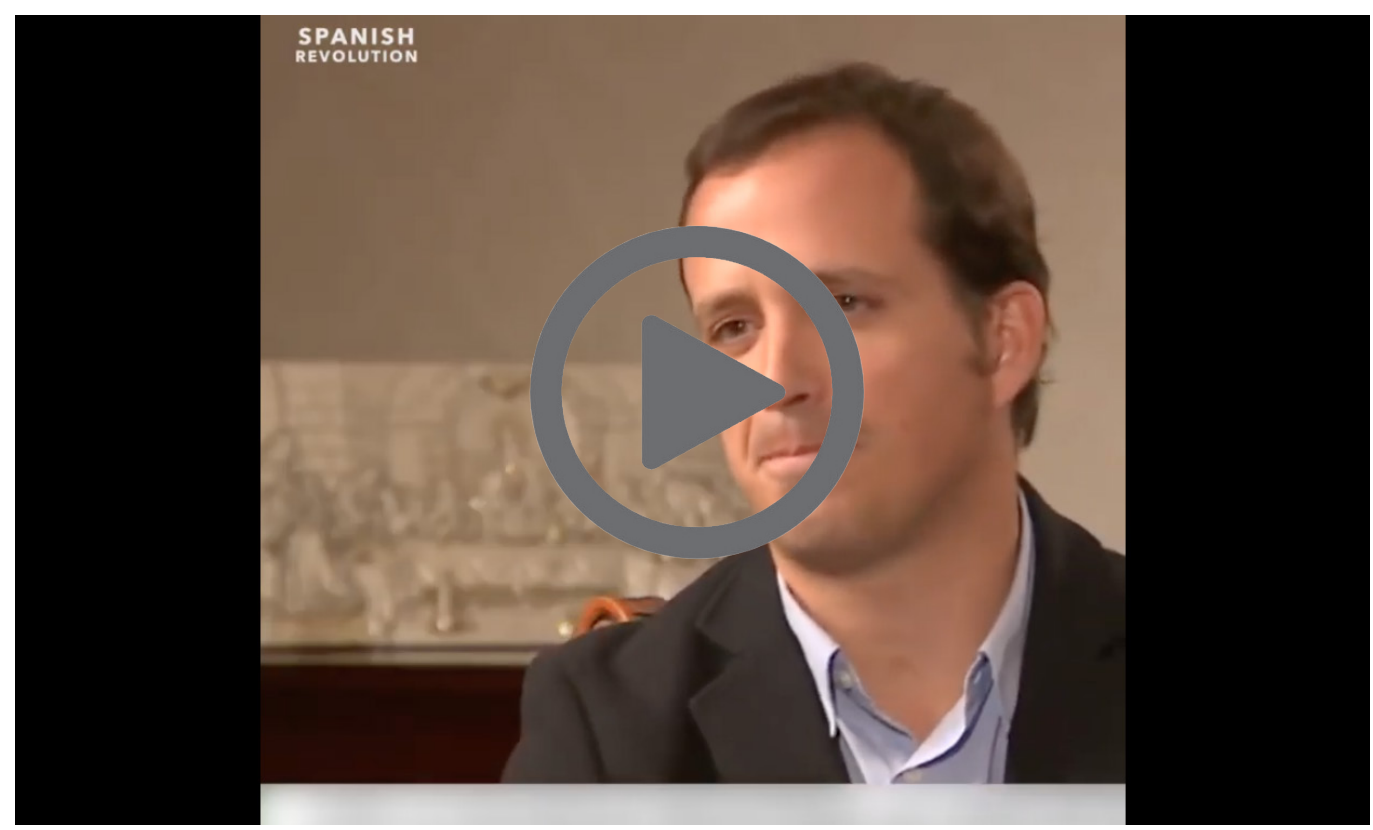


El tema se ha estudiado principalmente en Europa; desde hace cuarenta años aproximadamente, se identifica un grupo que se congregan alrededor del Laboratorio de Estudios sobre Convivencia y Prevención de la Violencia, ubicado en la Universidad de Córdoba en España. Estos estudiosos privilegian la línea de investigación psicoeducativa que "articula el conocimiento logrado mediante el trabajo científico a la intervención pedagógica rigurosamente realizada y evaluada" (Ortega, 2010, p.22).

Dicho grupo identifica tres momentos en que especialistas e investigadores se han interesado por el tema. El primero se refiere al descubrimiento del fenómeno como asunto de interés científico. El segundo tiene que ver con la definición del problema y la construcción de un lenguaje común con sentido unívoco, lo que generó, principalmente, estudios descriptivos. El tercero, de los años noventa a la fecha, fue cuando se realizan estudios explicativos con énfasis en la psicología educativa y el análisis de las características de los participantes como agresores, espectadores o víctimas.

En el año 2014, la Comisión de Derechos Humanos del Distrito Federal (CDHCM)

convocó a un diálogo público sobre violencia escolar y derechos humanos, a través de foros y un seminario interinstitucional e interdisciplinario con la participación de instancias de gobierno de los diversos niveles, de la academia, de organismos públicos de derechos humanos, de Organizaciones de la Sociedad Civil y comunidades educativas, reconociendo y estableciendo los canales adecuados para asegurar la participación directa y efectiva de niñas, niños y adolescentes en el mismo (CDHCM, 2014, p.1).

Pese a estos esfuerzos que consideran a tres actores sociales fundamentales gobierno (comisiones y consejos), organización de la sociedad civil y academiael problema del acoso y la violencia escolar sigue creciendo, y reproduce patrones de relación e identidades machistas y de la cultura patriarcal. Además, este problema no se erradica en las escuelas, bachilleratos ni universidades.

Los estudios del

[...] análisis de los factores explicativos del bullying [en inglés acoso y hostigamiento], son bastante ricos, los trabajos que ahondan en la dimensión afectiva y moral como base del comportamiento agresivo son notables [...] así como aquellos que se hacen acerca de los procesos de interpretación del mundo social desde aproximaciones tanto del procesamiento de la información social (SIP) como desde la Psicología Narrativa [...]. Otra importante línea de investigación y que enlaza directamente con las ya mencionadas tiene que ver con la naturaleza proactiva o reactiva del fenómeno y la diferente conceptualización y caracterización de éste de acuerdo con esta diferenciación (Ortega, 2010, p.76).

Los intentos de comprensión del fenómeno ahondan en sus componentes, dinámicas, procesos de origen, reproducción o complicidad a través de marcos de referencia y paradigmas teórico-conceptuales diversos, con sólidas preguntas, y rigor en sus métodos y técnicas de estudio. De esta manera, se 
alcanzan conclusiones fundamentales para tomar decisiones y cambiar las prácticas y significados que se reproducen en las escuelas por sus distintos actores educativos.

Sin embargo, en el contexto mexicano, preguntas como ¿qué tanto y de qué maneras padres de familia, profesorado y autoridades escolares, así como funcionarios educativos, impactan en la solución y erradicación del acoso y la violencia escolar?, ¿cuáles son los canales institucionales de diálogo entre investigadores y funcionarios de la educación? y ¿quiénes y cómo son los responsables gubernamentales para ofrecer programas permanentes, efectivos de prevención y atención? no son atendidas por la investigación. Además, los estudios en español son pocos para comprender la situación de los jóvenes escolarizados y generalmente se hacen fuera de México.

Si profesores, autoridades escolares, funcionarios de la educación usaran lo que se genera mediante la investigación, entonces, el conocimiento serviría para comprender la complejidad de la situación que viven, para atender los retos que enfrentan en los centros escolares de manera fundamental, y diseñar y elaborar programas, estrategias, y acciones puntuales y efectivas.

\section{Acerca del acoso escolar en México}

El acoso escolar incluye actos de hostigamiento que expresan la agresión entre distintos actores educativos, tanto en relaciones verticales de autoridades o personas mayores a menores o estudiantes, como horizontales, entre compañeros o colegas.

El problema de la agresión y la violencia en las escuelas mexicanas se ha estudiado bajo distintas denominaciones: bullying, maltrato escolar, intimidación entre iguales y acoso escolar. Cuando la agresión se expresa como abuso de la fuerza o del poder se convierte en un acto de violencia. Se identifican varios estudios aislados que trabajan el tema en distintas universidades y asociaciones nacionales y desde diversas disciplinas: la salud, la administración y la educación.

Acerca del acoso escolar en México se han realizado pocas investigaciones como tal, pero los temas de indisciplina y la violencia escolar merecieron dos números completos en la Revista Mexicana Investigación Educativa (año 2005, vol. 10, números 26 y 27). Se observa que hay menos estudios regionales, como en el caso de la Ciudad de México.

Por un lado, se identifica el estudio etnográfico de Gómez Nashiki (2005) en dos primarias públicas de la Ciudad de México, que da cuenta de cómo la violencia se gesta, sostiene y reproduce en la escuela mediante relaciones discriminatorias. Por otro, Saucedo Ramos (2005) explica la caracterización negativa que se hace de los alumnos que acuden a las escuelas en turnos vespertinos. Y, por último, Prieto 
Quezada, Carrillo Navarro, y Jiménez Mora (2005) concluyen la incidencia de factores familiares, escolares y sociales en este fenómeno y llevaron a cabo un programa de intervención para desarrollar conciencia sobre el fenómeno entre estudiantes de nivel medio superior en un bachillerato de Jalisco.

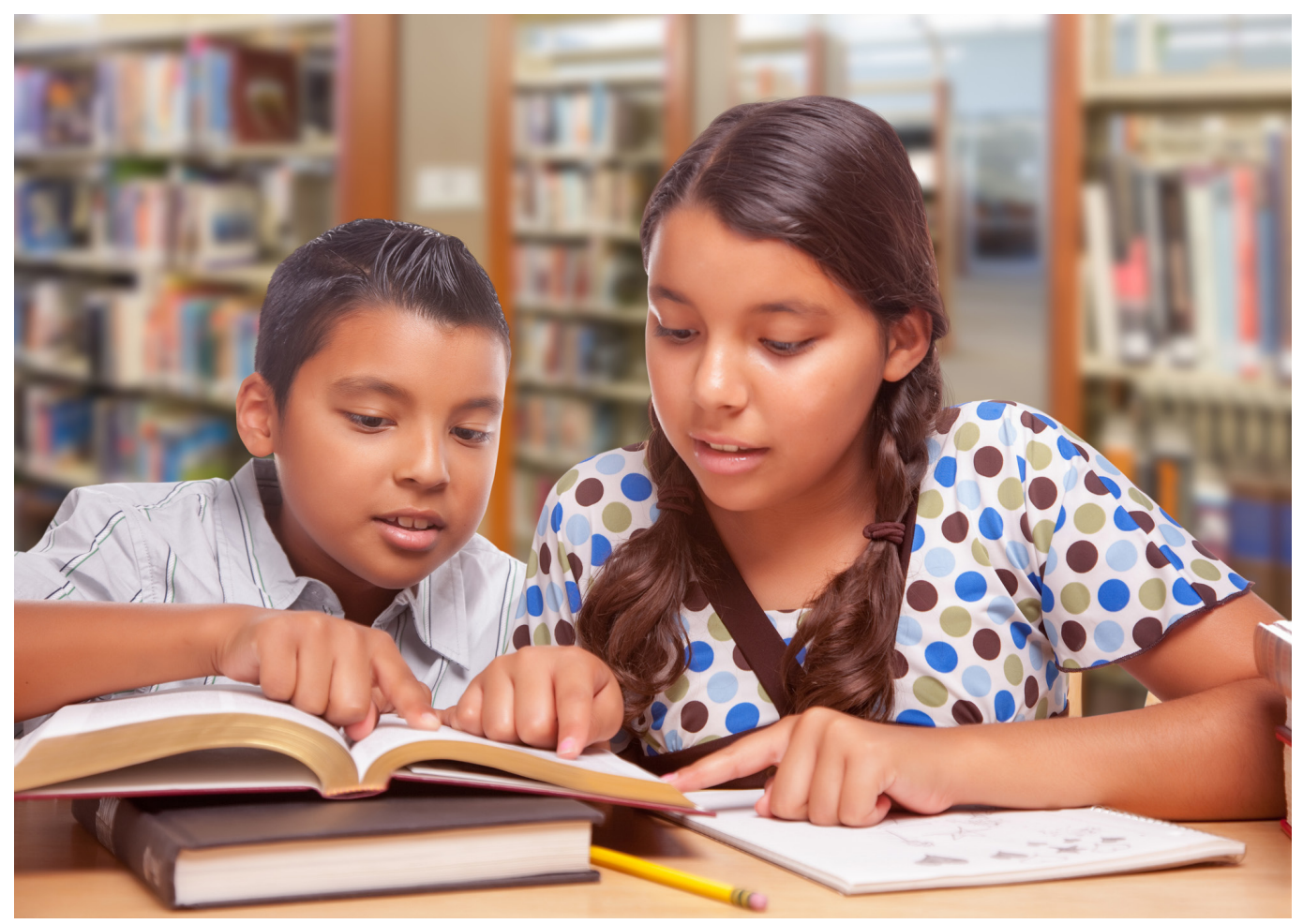

Además de la discriminación por el contexto familiar y socio-cultural de la familia de los niños, niñas y jóvenes, las creencias y prejuicios de las personas son los que dirigen sus acciones. Los estudios de

[...] algunos trabajos de corte cualitativo sobre el tema describen las percepciones de los maestros sobre la violencia escolar (Chagas, 2005). Saucedo (2005) explica la caracterización negativa que se hace de los alumnos que acuden a las escuelas en turnos vespertinos. Prieto et al. (2005) concluyen la incidencia de factores familiares, escolares y sociales en este fenómeno y llevaron a cabo un programa de intervención para desarrollar conciencia sobre el fenómeno entre estudiantes de nivel medio superior en un bachillerato de Jalisco. Por su parte, Vázquez et al. (2005) describen la percepción de los jóvenes sobre la violencia. A pesar de que todos estos trabajos constituyen un aporte a la caracterización de la violencia en las escuelas, aún hay mucho por investigar..." (Castillo Rocha y Pacheco Espejel; 2008, p. 826).

Por su parte, Vázquez Reyes (2005, p. 739) mediante un estudio en nueve preparatorias del Estado de México describe la percepción de los jóvenes sobre la violencia, tanto la ejercida por sus pares como la ejercida por los profesores. Con una muestra de 346 alumnos (193 mujeres y 153 hombres) se identificaron 205 episodios de intimidación entre pares y 228 sucesos violentos de los profesores. Estas percepciones y creencias también incluyen aquellas que tienen que ver con las pautas culturales acerca del género. 
Un estudio de Castillo Rocha y Pacheco Espejel, con estudiantes de secundaria en Mérida, Yucatán, describe el miedo escolar, la relación de los estudiantes con sus profesores, el abuso observado en las escuelas que padecen las víctimas y las diferencias de género. Observan que, aunque el maltrato verbal sigue siendo el de mayor frecuencia en ambos sexos, hay diferencias, pues los hombres parecen ser más insultados y las mujeres parecen ser víctimas de habladurías. Otra diferencia que se observó son las conductas de intimidación: los varones reciben más amenazas verbales y con armas que las mujeres. En general, los hombres padecen índices de abuso más elevados, por robo y golpes (Castillo Rocha y Pacheco Espejel, 2008, p. 837).

Es de tal forma que las prácticas y creencias de género, de clase social y del tipo de escuela van describiendo lo que se conoce como violencia psicológica, caracterizada, por ejemplo, por amenazas, calumnias, difamaciones, apodos, habladurías; y de lo que se define como violencia física, identificada, por ejemplo, por golpes, empujones, arañazos, sapes, jalones, pellizcos, nalgadas y hasta puñetazos o más.

Un estudio con pretensiones comparativas de los datos nacionales con los datos internacionales, es el de Muñoz Abundez, quien "analiza la magnitud de la violencia que reportan los alumnos de primaria y secundaria de México a través del informe Disciplina, violencia y consumo de sustancias nocivas a la salud en escuelas primarias y secundarias de México" (2008, p.1195), que presentó el Instituto Nacional para la Evaluación de la Educación (INEE), en comparación con las cifras relativas que ofrecen estudios de distintos países europeos, y otros dos, uno en Brasil y otro en Nicaragua.

Según Prieto Quezada et al. (2005), la violencia escolar tiene expresiones de violencia física en nuestro país y alcanza extremos tales como los secuestros exprés entre compañeros y la violación y el asesinato. Pero otro problema es la violencia de carácter institucional, que es una forma que se añade a las agresiones sufridas.

Los lugares donde se ejerce el acoso, el hostigamiento y la violencia ayudan a tipificar el problema. En suma: quien la ejerce y quien la padece, el lugar (por ejemplo, la casa, la calle, la escuela, etcétera), y las relaciones entre las personas (como compañeros de escuela, maestros y estudiantes, hermanos, padres e hijos, etcétera) ayudan a describir y entender la problemática escolar.

Acerca de la invisibilidad del acoso y la impunidad como una forma de agresión pasiva, Castillo Rocha y Pacheco Espejel (2008) comentan qué sucede con las autoridades educativas cuando finalmente los estudiantes y padres de familia manifiestan sus quejas por abuso. Lejos de escuchar e investigar las circunstancias, niegan el problema, protegen a los agresores y exponen a las víctimas. Se da el caso, por ejemplo, de quienes recomiendan al agredido y a sus familiares "no decir nada", "por su propio bien", pues el agresor "está muy bien parado en el sindicato" y puede generarse un ambiente adverso en otras escuelas por el simple hecho de haber denunciado el abuso. 
Estos investigadores también observan el maltrato en los medios de comunicación masiva, que frecuentemente exponen a las víctimas como si fueran mercancía de ratting (del inglés: delatar). Asimismo, comentan que los jóvenes están habituados a sufrir malos tratos y, frecuentemente, prefieren recibir un apodo humillante a ser ignorados por sus compañeros. Entonces, como sucedió en dicho estudio, después de señalar que los insultan, les pegan y los amenazan, los estudiantes comentan que se llevan bien. Esta aparente "normalidad" y la evaluación positiva de los estudiantes de secundaria sobre las relaciones sociales que se gestan en sus escuelas, lejos de ser una señal de "bienestar social", probablemente es un indicador de un proceso de socialización de la violencia, desde que se propone como algo que hay que tolerar, pues forma parte del ser habitual de las escuelas (Castillo Rocha y Pacheco Espejel, 2008, p. 839).

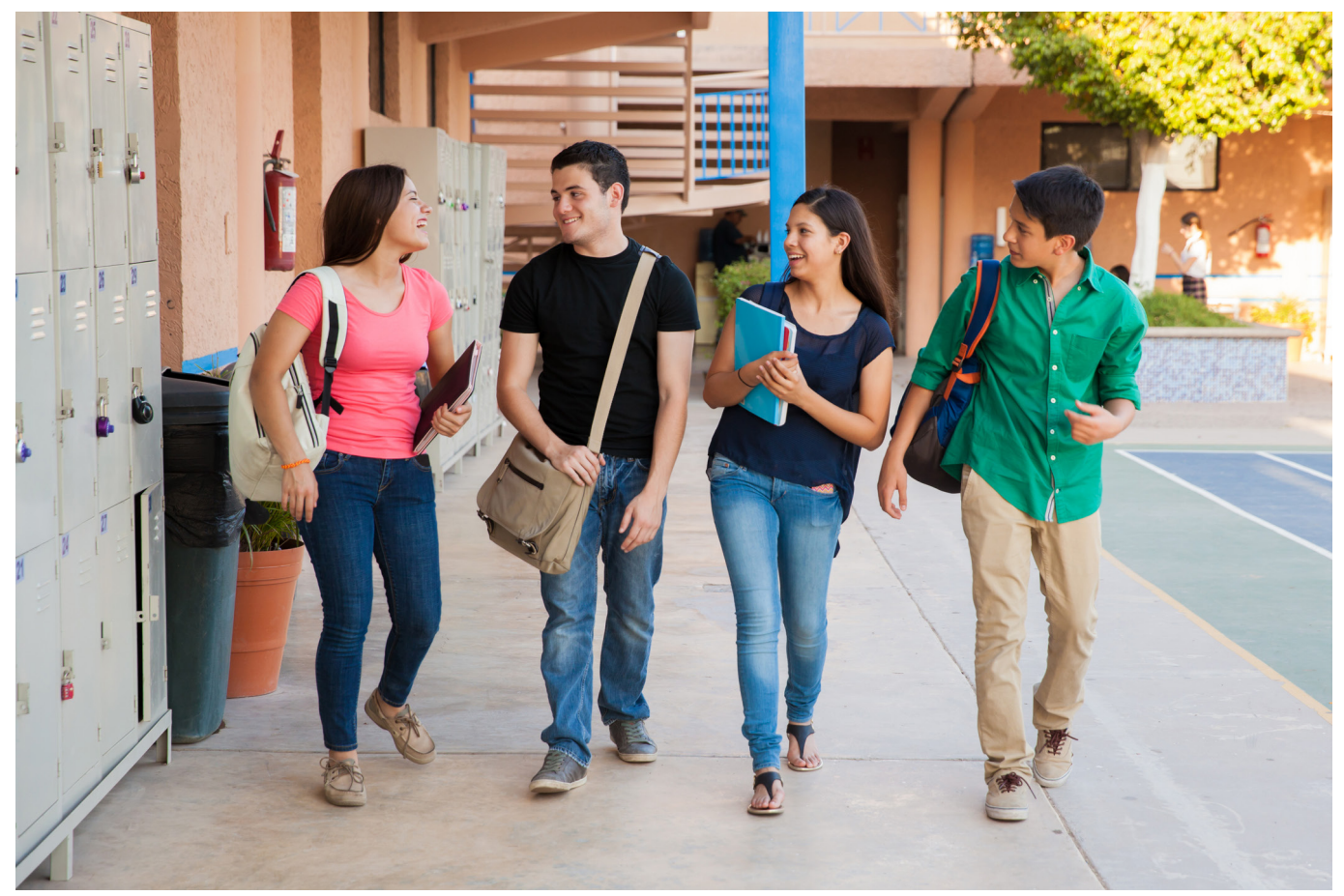

Con el conocimiento de que la violencia física, psicológica, sexual y la que se ejerce sobre las pertenencias, sucede en las escuelas, los estudios identificados mencionan poco el robo de ropa, comida, útiles escolares, dinero y, en general, las pertenecías personales de profesores y estudiantes. En caso de mencionarse se hace de manera que no se precisa o conceptualiza como violencia patrimonial.

\section{¿Cómo cambiar las prácticas del acoso y la violencia escolar?}

Al ser jóvenes entre los 12 y 15 años que cursan la secundaria, y de 15 a 19 el bachillerato, preguntarse acerca de las maneras en que es posible erradicar la violencia de las escuelas pretende invitar a iniciar la búsqueda en un contexto 
histórico y cultural de profundas desigualdades sociales y económicas generador de diferentes tipos de violencia y acoso escolar -, así como de formas y prácticas culturales muy arraigadas.

También está fijada la idea entre los actores educativos de reflexionar y estudiar el acoso como una dimensión ética atribuible al estudiante o al profesor, más que concebirla como una práctica social. Pensar que el acoso escolar tiene sólo una dimensión afectiva es individualizar el problema y llevarlo a las características intrínsecas de la persona, así se le reduce y simplifica a una dimensión. El acoso y la violencia escolares son procesos sociales con prácticas e interacciones construidas mediante contextos de relaciones entre estudiantes y profesores, en espacios y tiempos específicos.

Todo tipo de exclusión provoca violencia. Una manera de concebir a los niños, niñas y jóvenes, así como a los profesores, desde la lupa de la violencia, hace una parada en la desigualdad social, la discriminación y los prejuicios sociales desde lo colectivo, y en la idea de los efectos del desamparo y el abandono familiar y social — de padres, maestros y directores - que viven algunos niños, niñas y adolescentes. Por ejemplo, los niños y jóvenes que viajan a los campos agrícolas con sus padres como jornaleros, de quienes nacen y viven en los sistemas carcelarios, de aquellos que están bajo la tutela del gobierno en casas hogares y albergues escolares, u otras situaciones de vida en internamiento.

Estos contextos de desamparo infantil y juvenil se evidencian en las huellas que dejan en el cuerpo y la mente — desamparo subjetivo- de los estudiantes. Las prácticas de violencia que se expresan en formas de desamparo como el hambrey la desnutrición, por pobreza o desatención de padres y maestros, tienen consecuencias imborrables como el desmedro y la emaciación. El desamparo deja huellas de los ultrajes al cuerpo por los abusos sexuales y de lesiones físicas, consecuencia de castigos e impulsividad de los adultos. El desamparo se imprime en la mente de las niñas, los niños y los jóvenes como algo intangible, un tanto invisible en sus emociones y vergüenza (Bautista Hernández et al., 2019); temas poco estudiados como violencia contra de la infancia y la adolescencia.

El problema de la desigualdad e inequidad social es una condición que propicia actos de violencia, que necesitan ser abordados como parte del marco de referencia de la vida y experiencia subjetiva, y de la construcción de significados de los jóvenes que experimentan acoso escolar (Reguillo, 2010). Los estudios de la sociología de la violencia (Debarbieux y Blaya, 2010) muestran cómo los centros educativos tienen una importante influencia en la construcción de la violencia.

Hay que distinguir entre la violencia del dominio social, como un abuso del poder en las relaciones interpersonales, y la agresión inherente de la infancia y del ser humano, como un mecanismo de supervivencia. Así, se abre el debate entre la dimensión endógena y la exógena de la violencia y la agresión. La agresión 
Video 2. La violencia: qué la genera y qué la previene | Feggy Ostrosky | TEDxYouth@ BosquesDeLasLomas como una conducta de respuesta de protección que busca la supervivencia y la defensa no es intencional ni deliberada, es biológica e instintiva, tiene diferentes manifestaciones y no tiene dirección específica. En contraste, la violencia es una construcción social que desestructura, es destructiva, intencional, direccionada para controlar, someter, dominar; o sea, la diferencia sustantiva está en el abuso de poder (Naranjo, 2013; Freedman y Stoddard Holmes, 2003, ver video 2).

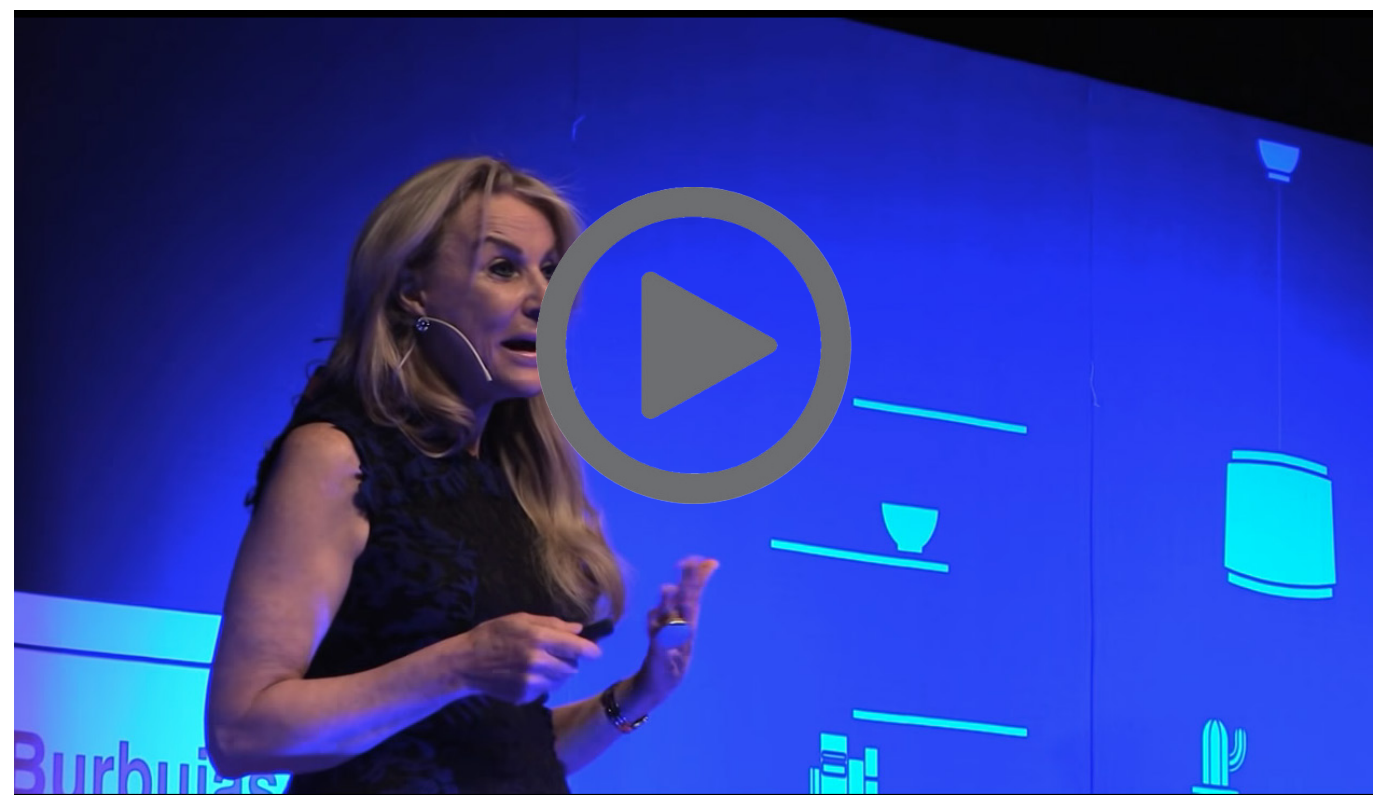

Ahora bien, la escuela cuenta con la posibilidad de tejer, a través de la cultura, una malla que ampare al sujeto y al colectivo, que promueva la estructura de relaciones sociales de buen trato, cuidado y bienestar. La escuela puede ser el espacio de relaciones sociales de otro orden: el racional, en el que se construyan relaciones sociales de acuerdo con concepciones, creencias, tradiciones, usos y costumbres de convivencia, creación, equidad, dignidad y libertad a través del entendimiento y el diálogo.

Este reconocimiento en las escuelas puede abrir paso al conocimiento con una filiación a una comunidad (Wenger, 1999), que permita el aprendizaje y la construcción de identidades solidarias, empáticas y propicias para el desarrollo de los niños, niñas y jóvenes; y, como efecto de los dos, abrir espacios para un proceso de filiación cultural. Sin ambas, la pertenencia cultural y el proceso de filiación institucional se dificultan y, entonces, los procesos de identidad social y psicológica se ven trastocados. Se desconoce cómo el acoso escolar altera el proceso de filiación institucional como parte de la construcción de la propia identidad y del aprendizaje social. Es necesario explorar al respecto en México.

Parece ser que hasta el año 2014 la investigación abordó los tipos, contextos y prácticas sociales, y los actores de acoso y violencia escolares. A partir de estos estudios se comienzan a investigar otros temas como las interacciones 
y la multicausalidad del fenómeno, así como las conexiones de las percepciones socioculturales, los mensajes explícitos e implícitos, y las conductas de los padres y maestros (Ruvalcaba Romero et al., 2015), tanto agresivas como prosociales y de buen trato.

Surge, también el estudio de otra forma de violencia escolar —y social—, el de la homofobia como:

el rechazo a las personas o los actos que no siguen el modelo heterosexual hegemónico o que en su actuar cotidiano revelan actitudes sexuales o genéricas diferentes a las establecidas para lo masculino y lo femenino desde los marcos normativos de género, a partir de prácticas que pueden ir desde el desdén, pasando por la injuria hasta actos de agresión que pueden llevar al asesinato (List Reyes, 2016).

Otro tema más reciente es el de violencia de género impartida por las instituciones escolares. Por ejemplo, la violencia simbólica al omitir, negar o invisibilizar el acoso, la violencia en redes sociales, en lo que se considera culturalmente lo femenino y lo masculino, determinando los actos de erotismo, y el sexting, mediados por las tecnologías y las redes sociales (Gálvez Antúnez, 2018), con prácticas en las que los y las jóvenes se envían fotografías y videos de contenido sexualmente explícito, a través de dispositivos inteligentes y teléfonos celulares.

En suma, el cambio es una estrategia de dos puntas. Una es la búsqueda de políticas y acciones hacia la equidad social y económica, y la igualdad política, que contribuyan de manera fundamental a que en los centros escolares se cambien las prácticas y contextos sociales de acoso por otras de ciudadanía en el diálogo, buen trato y educación de los actores educativos. La otra será hacia todas aquellas políticas y acciones sociales - de gobierno, familiares y comunitarias - de protección y amparo de la infancia y la juventud; es decir, de construcción de ciudadanía.

\section{Conclusiones}

El acoso y la violencia escolar son temas de interés científico y relevancia social y educativas. La violencia no es solamente un hecho o fenómeno, sino que se expresa a través de actos, el violento y el discursivo. Es decir, las maneras de hablar sobre algunos actos muestran el significado que construyen los actores educativos acerca del acoso, el hostigamiento y la violencia. Así, todo se convierte en el acto violento. Los actos violentos de los actores educativos son un elemento más en las relaciones escolares: entre alumnos, docentes, familias y autoridades escolares.

Hoy en México se estudian los contextos, percepciones, discursos y prácticas escolares de acoso y violencia, que se alejan de la concepción individual y psicológica del problema, y la conciben como un fenómeno social multifactorial, multicultural y multicausal. 
A pesar de que hay estudios con métodos cuantitativos y cualitativos, éstos no consiguen ofrecer diagnósticos que permitan comprender el problema y su ubicuidad mediada por los contextos de relaciones sociales, su dimensión histórica y cultural, y su estrecha relación con diversas cosmovisiones y éticas, que forman parte del multiculturalismo de México.

Por lo tanto, la escuela participa en la reproducción cultural y social del acoso y de la violencia, con formas abiertas y naturalizadas de relación y convivencia de los distintos actores educativos. Los resultados, a través de la evidencia fundamental de la literatura científica acerca de la relación que existe entre la victimización y la agresión, y el clima escolar, así como de la construcción de identidades e inequidad de género, suceden en contextos sociales imbricados, tanto con factores materiales y organizativos como humanos.

\section{Referencias}

* Bautista Hernández, G., Vera Noriega, J. A. y Tánori Quintana, J. (2019). Shame, Empathy, Coping and School Safety of the Bystanders in Situations of Bullying. Trends in Psychology, 27(2), 357-369. Dol: http://dx.doi.org/10.9788/tp2019.2-05

* Castillo Rocha, C. y Pacheco Espejel, M.M. (2008, julio-septiembre). Perfil del maltrato (Bullying) entre estudiantes de secundaria en la Ciudad de Mérida, Yucatán. Revista Mexicana de Investigación Educativa, 13(38), 825-842. https://www. redalyc.org/articulo.oa?id=14003807

* Díaz-Barriga Arceo, F., Vázquez-Negrete, V. I., y Díaz-David, A. (2019). Sentido de la experiencia escolar en estudiantes de secundaria en situación de vulnerabilidad. Revista Latinoamericana de Ciencias Sociales, Niñez y Juventud, 17(1), 237-252. Dol: https://dx.doi.org/10.11600/1692715x.17114

* Chagas Dorrey, R.C. (2005, octubre-diciembre). Los maestros frente a la violencia entre alumnos. Revista Mexicana de Investigación Educativa, 10(27),1071-1082. https://www.redalyc.org/articulo.oa?id=14002706

* Comisión de Derechos Humanos de la Ciudad de México (CDHCM). (2014, 10 de junio). Convocan CDHDF, académicos y osc a atender violencia escolar de manera integral [boletín 107/2014]. https://cdhcm.org.mx/2014/06/convocan-cdhdfacademicos-y-osc-a-atender-violencia-escolar-de-manera-integral/

* Debarbieux E. y Blaya, C. (2010). Sociología y violencia escolar. Un enfoque contextual. En R. Ortega (Coord.), Agresividad Injustificada, Bullying y Violencia Escolar (pp. 355-383). Alianza Editorial.

* Dorado, J. (2019, 9 de abril). Cambiar la educacion para cambiar el mundo: Claudio Naranjo [video]. YouTube. https://www.youtube.com/watch?v=krOPXVRNMIY

* Freedman, D. P.y Stoddard Holmes, M. (Eds.). (2003). The Teacher's body. Embodiment, Authority, and Identity in the Academy. State University of New York Press. 
* Gálvez Antúnez, A. L. (2018, julio-diciembre). El sexting, del erotismo a la violencia institucional: dos casos en adolescentes de secundaria, Ethos Educativo, 53, 7-33. http://www.imced.edu.mx/Portal/files/Ethos/Archivo/53/53-1.pdf

* Gómez Nashiki, A. (2005, julio-septiembre). Violencia e institución educativa. Revista Mexicana de Investigación Educativa, 10(26), 693-718. https://www.redalyc. org/articulo.oa?id=14002605

* List Reyes, M. (2016, enero-junio). Los universitarios frente a la homofobia. El caso de la Benemérita Universidad Autónoma de Puebla. Sinéctica, Revista Electrónica de Educación, 46, 1-15. https://www.redalyc.org/articulo.oa?id=99843455003

- Naranjo, Claudio. (2013). Cambiar la educación para cambiar el mundo. Ediciones La Llave.

* Muñoz Abundez, G. (2008). Violencia Escolar en México y en otros países. Comparaciones a partir de los resultados del Instituto Nacional para la Evaluación de la Educación. Revista Mexicana de Educativa, 13(39), 1195-1228. https://www. redalyc.org/articulo.oa?id $=14003908$

* Ortega, R. (Coord.). (2010). Agresividad Injustificada, Bullying y Violencia Escolar. Alianza Editorial.

* Prieto Quezada, M.T., Carrillo Navarro, J.C. y Jiménez Mora, J. (2005, octubrediciembre). La violencia escolar un estudio en el nivel medio superior. Revista Mexicana de Investigación Educativa, 10(27),1027-1045. https://www.redalyc.org/ articulo.oa?id=14002704

Reguillo, R. (Coord). (2010). Los jóvenes de México (1er ed.). FCE- ConACuLTA.

* Ruvalcaba Romero, N. A., Murrieta Cummings, P., Rayón Carlos, R. y Pimentel Pinedo, A. (2015). Parental approval of violence and victimization as predictors of aggression and prosocial behavior. Tesis Psicológica, 10(1), 60-71. https://www. redalyc.org/articulo.oa?id=1390/139044651005

* Saucedo Ramos, C.L. (2005, julio-septiembre). Los alumnos de la tarde son los peores. Prácticas y discursos de posicionamiento de la identidad de alumnos problema en la escuela secundaria. Revista Mexicana de Investigación Educativa, 10(26), 641-668. https://www.redalyc.org/articulo.oa?id=14002603

* TEDx Talks. (2015, 25 de agosto). La violencia: qué la genera y qué la previene | Feggy Ostrosky | TEDxYouth@BosquesDeLasLomas [video]. YouTube. https:// youtu.be/KwGEIFJgB7g

* Vázquez Valls, R., Villanueva Mercado, A. E., Fernando Rico, A. y Ramos Herrera, M. A. (2005, octubre-diciembre). La comunidad de la Preparatoria 2 de la Universidad de Guadalajara. Actitudes de sus miembros respecto de la violencia y no violencia escolar. Revista Mexicana de Investigación Educativa, 10(27), 1047-1070. https:// www.redalyc.org/articulo.oa?id=14002705

* Velázquez, Reyes, Luz María. (2005, julio-septiembre). Experiencias estudiantiles con la violencia en la escuela. Revista Mexicana de Investigación Educativa; 10(26), 739-764. http://www.scielo.org.mx/pdf/rmie/v10n26/1405-6666-rmie-10-26-739.pdf 
* Wenger, E. (1999). Comunidades de práctica. Aprendizaje, significado e identidad. Paidós.

\section{Cómo citar este artículo}

* de Agüero Servín, Mercedes. (2020, julio-agosto). La investigación acerca del acoso y violencia escolares en México. Revista Digital Universitaria (RDU), 21(4). DOI: http://doi.org/10.22201/cuaieed.16076079e.2020.21.4.2

Recepción: 28/04/2020. Aceptación: 01/06/2020 